\title{
New advances in the field of planetary nebulae from ultraviolet observations
}

\author{
Luciana Bianchi \\ Johns Hopkins University, Dept. of Physics and Astronomy \\ 3400 N. Charles St. Baltimore, MD 21218-2695, USA \\ email: bianchi@pha.jhu.edu
}

\begin{abstract}
The ultraviolet (UV) domain, in particular shortwards of $\operatorname{Ly} \alpha$, provides unique information to unravel the physical parameters of Central Stars of Planetary Nebulae (CSPNe) and the paths for this elusive final stage of stellar evolution, thanks to a wealth of diagnostic transitions from ionic species not observable at other wavelengths. Intermediate mass stars are the major providers of important elements like $\mathrm{C}$ and $\mathrm{N}$. Understanding how they shed most of their initial mass is critical for understanding the chemical enrichment of the ISM. Mass-loss diagnostic lines abound at UV wavelengths, and when the CSPN reaches the hottest $T_{\text {eff }}$ before turning on the WD-cooling sequence, and the wind fades, the last wind lines to disappear are found in the far-UV, as well as diagnostic lines for elements such as Ne. This domain also offers a host of $\mathrm{H}_{2}$ transitions, tracing the circum-stellar material expelled in previous phases. UV images and spectra of PNe add critical constraints to their ionization structure and to some abundances. Finally, the recent GALEX sky surveys in two UV bands afforded the first unbiased census of hot white dwarfs (WD) and post-AGB objects in the Milky Way, significantly expanding known catalogs and providing statistical constraints to the initial-final mass relation.
\end{abstract}

Keywords. stars: AGB and post-AGB, stars: atmospheres, stars: abundances, stars: fundamental parameters, stars: mass loss, stars: winds, outflows, white dwarfs, planetary nebulae: general, Galaxy: stellar content, ultraviolet: stars, surveys, catalogs

\section{Introduction}

In the first part of the Symposium, progress in the field of PNe was reviewed according to observing technique, or rather, by wavelength range, and the welcome task fell on me to summarize results primarily enabled by ultraviolet (UV) data.

Planetary Nebulae, a short-lived and quite eventful phase of intermediate-mass stars' evolution, offer valuable insight into their progenitors' previous evolution, final fate, and contribution to the chemical enrichment of the interstellar medium (ISM) through the yield of nucleosynthesis products. After most of the mass has been shed during the main sequence and AGB phases, the central star becomes hot enough to ionize the surrounding envelope, suddenly "illuminating" the pages of the book that tells the star's story. The chemical abundances of the CSPN, and of the surrounding layers, reveal the star's past structure and nucleosynthesis history (Kwitter 2012, Marigo et al. 2001).

The powerful UV radiation from the very hot CSPN also accelerates a radiationpressure driven wind, whose supersonic velocity (albeit low density) carries enough momentum to plow the slower, denser AGB-originated outer envelope, building up a dense shell at the shock front (first described by Kwok 1987). The hot central star's radiation and the shock energy ionize the nebula making it easy to detect and study.

The PN and its central star are a complex system, packed with information. Its study involves the hottest stars' atmosphere physics, stellar winds, shocks and outflows with complex [magneto]-hydrodynamic phenomena (including highly collimated outflows, and 




Figure 1. Wind lines abound in the far-UV for very hot stars. Model spectra are shown for $T_{\text {eff }}$ decreasing from top to bottom (with typical values for $\dot{M}$ and other parameters). At the hottest $T_{\text {eff }}$, OVI and NeVII are prominent wind lines, while diagnostics at longer wavelengths fades away (models from the grid of Herald \& Bianchi 2011), computed with the CMFGEN code (Hillier \& Miller 1999; see also Keller et al. 2011

possibly disks), the evolving shape and speeds of the bubbles encoding the intertwined characteristics of the outflows from AGB, late-AGB, and CSPN (e.g. Kwok 2003; Balick 2012; Manchado et al. 2012 and references therein), the ionized PN gas which reflects the chemical composition of the former atmosphere and the radiation of the current one, and neutral and molecular gas, accounting altogether for the initial stellar mass.

For each of these components, a great deal of information is revealed at ultraviolet wavelengths. The number of transitions [per wavelength interval] from abundant ions in the interstellar- or PN-circumstellar- medium is much higher in the UV than at other wavelengths; most, and most crucial, diagnostic lines for the hottest CSPN and their winds fall in this domain.

In this paper we highlight the major UV-specific contributions to recent advances in the above areas, and future outlooks. For our purpose, let's first define the UV range as the portion of the electromagnetic spectrum shortwards of $\approx 3200 \AA$, the cut-off of $\mathrm{Cs}_{2} \mathrm{Te}$-photocathode detectors with $\mathrm{MgF}_{2}$ window. Their range, extending just shortwards of $2000 \AA$, is usually termed near-UV (NUV). Shorter wavelengths (far-UV, FUV) are observed e.g.with CsI-cathode cross delay line (XDL) detectors (e.g. GALEX FUV,

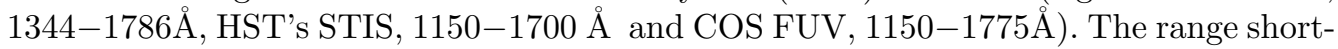
wards of Ly $\alpha$ is harder to observe with good sensitivity, but of utmost importance. The Far-Ultraviolet Spectroscopic Explorer (FUSE) covered the 905-1187 A range at spectral resolution of $R \approx 20,000$, with a 10,000 -fold increase in sensitivity over its predecessor, Copernicus (1970's), and observed over 3,000 science objects, from 2000 to 2007, including over forty Galactic CSPN with wind lines. 


\section{The central star: Peeking into the core of the past, and into the PN engine}

Great strides have been accomplished in modeling CSPN spectra, thanks to (i) the observation of new diagnostic lines in the FUSE range, which -combined with lines at longer wavelengths- enabled the many photospheric and wind parameters to be uniquely constrained, and (ii) advances in model calculations. We will mention some results, which opened new paths still to be fully exploited.

For the CSPN approaching the hottest $T_{\text {eff }}$ 's in the constant-luminosity track (before turning into the WD cooling track), diagnostic lines are scarce, but the OVI $\lambda \lambda 1031.9,37.6$ doublet and NeVII $\lambda 973$ appear as conspicuous P Cygni profiles in their spectra (Fig.1). Their strong $\mathrm{P}$ Cyg line observed at $\sim 973 \AA$ had been previously mis-identified with CIII $\lambda$ 977, which is prominent in cooler CSPNe, as shown also by the models in Fig.1bottom, and in massive stars (e.g. Bianchi \& Garcia 200; Garcia \& Bianchi 2004). But in the hottest $\left(T_{\text {eff }} \gtrsim 80,000 \mathrm{~K}\right) \mathrm{CSPN}$, no model which could explain their hotter lines would predict CIII to be a strong P Cyg. The CIII mis-identification also caused the wind terminal velocity to be largely overestimated $\left(\gtrsim 4000 \mathrm{~km} \mathrm{~s}^{-1}\right)$. Including ions up to NeVIII in model calculations led to the correct identification of this line with NeVII $\lambda 973$. Models also showed that NeVII $\lambda 973$ is very sensitive to the Ne abundance (Bianchi \& Herald 2007, Herald et al. 2005) whose value can be constrained when other physical parameters such as $\dot{M}$ are independently derived (Fig.2). The Ne abundance is an important test to theories of post-AGB evolution, in particular to the occurence and effects of the VLTP, invoked as a possible explanation for H-deficient CSPNe. In the "born-again" scenario, as the star in the hot pre-WD phase returns to the AGB (triggered by a He-shell flash, Herwig 2005) and then travels the CSPN track again, Ne overabundance is predicted. Spectral analyses found Ne to be overabundant in H-poor CSPN (Bianchi \& Herald 2007) as well as in PG1159 stars (Werner et al. 2004), in line with theoretical predictions, and about solar in H-rich CSPNe (Herald \& Bianchi 2011). The NeVII $\lambda 973$ wind line, when not saturated, is also very sensitive to $\dot{M}$ (Bianchi \& Herald 2007).

Other abundances relevant for stellar evolution theories include Ar (Keller et al. 2011 and references therein), and Fe. In H-deficient CSPNe, iron is expected to be underabundant; actual measurements are difficult for hot stars, and results puzzling, see Werner et al. (2010), Keller et al. (2011). Fe lines may also be affected by clumping (Fig. 3). Ultimately, we wish to compare these tell-tale element abundances in CSPN with their PNe (but samples with existing measurements scarcely overlap), and with their presumed descendants, hot WDs and PG1159 stars (e.g. Jahn et al. 2007, Werner 2012).

Another important fact to reckon in modeling CSPNe is that the wind is not homogeneous but clumped. Clumping was postulated early on, as inherent to the mechanism of radiation-pressure acceleration, which is prone to generate inhomogeneities and highervelocity, denser regions. The effect of clumps in hot stars' winds began to be explored over a decade ago (e.g. Hamann \& Koesterke 1998; Hillier \& Miller 1999). Observationally, constraining the degree of clumping (parametrized as a filling factor $f=1 / D$ where $D$ is the density enhancement in the clumps with respect to a smooth-wind) rests on the fact that resonance lines with linear dependence on density $\mathrm{n}_{e}$ would not be affected by clumping in the same way as $\mathrm{H} \alpha$ in $\mathrm{O}$ stars, and other emission lines in W-R (e.g. Kudritzki et al. 2006; Urbaneja et al. 2006). Indeed, models with clumping can fit all diagnostic lines which otherwise call for discrepant physical parameters. When there is sufficient diagnostics, the $f$ value can be constrained and a unique solution for the physical parameters $\dot{M}, T_{\text {eff }}$, etc. can be derived, for hot massive stars and for CSPNe alike (e.g. Bianchi et al. 2009; Hamann 2008; Georgiev et al. 2008; Herald \& Bianchi 2011). 

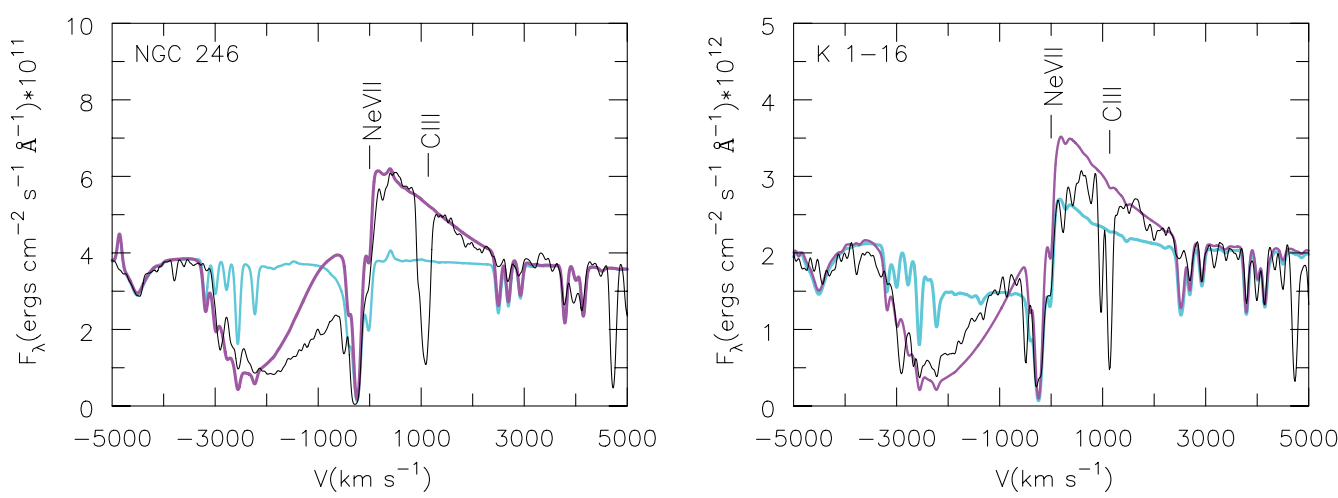

Figure 2. NeVII $\lambda 973$ in the FUSE spectrum (black) of NGC $246\left(T_{\text {eff }}=165 \mathrm{kK}\right)$ and K1-16 $\left(T_{\text {eff }}=135 \mathrm{kK}\right)$; CMFGEN models with solar and $10 \times$ solar abundance shown in cyan $/$ pink (light/dark grey), with absorption by ISM $\mathrm{H}_{2}$ applied. From Bianchi \& Herald (2007)


Figure 3. Effects of wind clumping on PV $\lambda \lambda 1118 / 28$ (left, STIS spectrum of NGC 6543) and FeVI lines (right, FUSE spectrum of IC 4593). CMFGEN models are shown for different clumping filling factors ( $f=1$ means no clumping). Figure from Herald \& Bianchi (2011).

Lines are most sensitive to this effect when they are not saturated, such as PV $\lambda \lambda 1118 / 28$. Typically, values of $0.04 \leqslant f \leqslant 0.1$ are found, implying lower mass loss rates by a factor of $\sim 3-5$ with respect to a smooth-wind model, as $\dot{M}$ (clumped) $=\sqrt{f} \times \dot{M}$ (smooth). The importance of the result is twofold: first, inclusion of clumping enables all diagnostic lines to be fitted consistently, removing the ambiguity on $\dot{M}$ from different diagnostics and better constraining physical parameters. Second, insights on the wind are gained. A precise derivation of $\dot{M}$ is relevant for the CSPN evolution and PN dynamical evolution. These recent results are based on the micro-clumping approximation (clumps smaller than the photons' mean free path). Macroclumping has also been considered, whereby the derived $\dot{M}$ may not be reduced (Hamann et al. 2008; Oskinova et al. 2007).

Clumps also produce X-rays from shocks in the wind. Again first recognized in massive stars (e.g. Bianchi \& Garcia 2002) this has a relevant effect on the wind ionization (necessary to explain the "super-ions", such as OVI) when $T_{\text {eff }} \lesssim 50,000 \mathrm{~K}$.

The major issue that hampers our understanding of CSPN evolution is the uncertainty in their distance; this is overcome if we can observe objects in other galaxies with sufficient resolution for a detailed analysis. Among the dimmest objects observed with FUSE were 12 LMC and 3 SMC CSPN: the advantage of the known distance led to "convincing" mass estimates between 0.55 and $0.65 M_{\odot}$ (Herald \& Bianchi 2004a, 2007; see also discussion by Kudritki et al. 2006 and comparison with MW stars). Another advantage of studying 

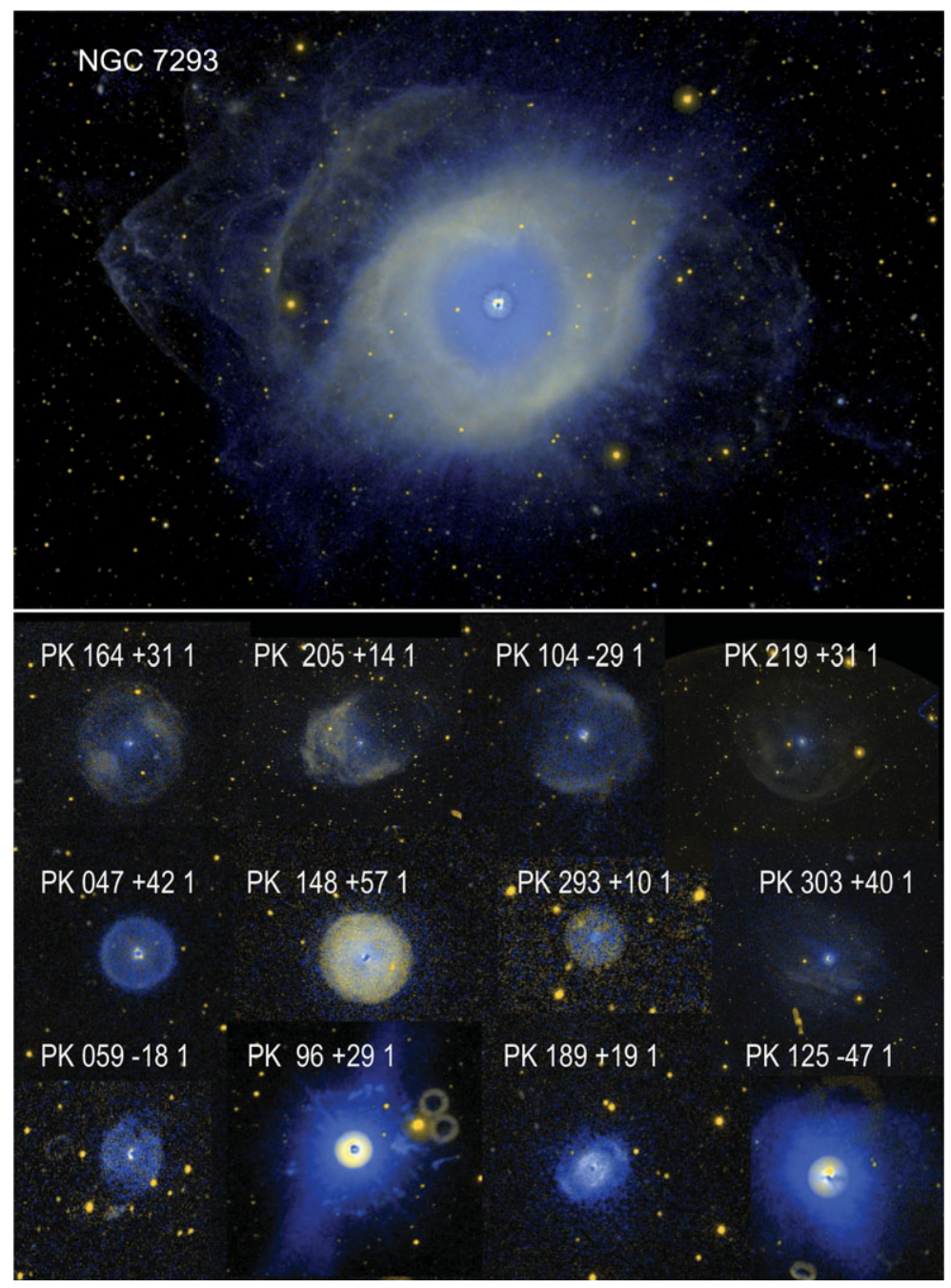

Figure 4. GALEX color-composite images of a sample of PNe: FUV in blue, NUV in yellow (the yellow dots are MW foreground/background cool stars, not emitting in FUV). Examples are shown of PNe with ansae (typically yellower than the inner region), some spherical objects with varying FUV/NUV ratios (the "Owl" structure is not visible in the representation, where we kept a linear stretch in all images, but it is well visible with different intensity stretch), some evolved objects and some with strong FUV emission. The central star is saturated in most of the famous objects (in some of the examples shown, NUV is saturated to the extent of producing nearby ghosts in the form of yellow rings, and a round "skirt" from the psf tail).

objects in a different galaxy is to explore effects of metallicity. We found in our MC sample wind terminal velocities $V_{\infty} \sim 700-1300 \mathrm{~km} \mathrm{~s}^{-1}$, about half the typical MW values, and $\dot{M} \sim$ few $\times 10^{-8} \mathrm{M}_{\odot} \mathrm{yr}^{-1}$ in most cases (higher in two objects).

\section{The planetary nebulae ionized shells at UV wavelengths}

Imaging and spectroscopy at various wavelengths combined is needed to interpret the structure of PNe (Peimbert 1978, Torres-Peimbert \& Peimbert 1997, Kwok 2010).

Unraveling the PN composition is important because its chemical abundances yield clues to evolutionary origins, and its ionization structure conveys information on the 




Figure 5. Hot and cool $\mathrm{H}_{2}$ in A $35 \mathrm{Ly} \beta$ region. The observed spectrum (red/light grey) was fitted with a TLUSTY model $\left(T_{\text {eff }}=80,000 \mathrm{~K}\right)$, to which the effects of two $\mathrm{H}_{2}$ components are applied. The best-fit model is shown with "cool" (ISM-like) $\left.\mathrm{H}_{2} \quad\left(\log N_{\mathrm{i}}\right)=19 \mathrm{~cm}^{-2}, T=200 \mathrm{~K}\right)$ applied (green), which matches some of the narrow absorptions but leaves others unexplained, and with a small additional hotter component (blue) with $\left(\log N_{\mathrm{i}}\right)=17.4 \mathrm{~cm}^{-2}, T=1250 \mathrm{~K}$ (figure adapted from Herald \& Bianchi 2002).

ionizing source (the central star) and reflects the density and dynamical structure of the nebula. Observed kinematics and morphology are the combined product of the AGB wind and the CSPN wind interacting, their often asymmetric density structures and complex dynamics leading to the variety of $\mathrm{PN}$ morphologies projected in $2 \mathrm{D}$ onto the sky. As for the PN ionization and abundances, the UV domain includes several relevant transitions (e.g. Shaw et al. 2010; Milingo et al. 2006). Here we will only recall that the GALEX UV sky surveys covered most of the sky with FUV (1344-1786 $\AA$ ) and NUV (1771-2831 $)$ wide-field imaging, at depths of $\sim 27.5 \mathrm{mag} / \mathrm{sq} . \operatorname{arcsec}$ (MIS survey, $\sim 2000$ sq.deg.) and $\sim 25.5 \mathrm{mag} / \mathrm{sq}$.arcsec (AIS survey, >28,000 sq.deg.), plus deep fields, see Bianchi (2009). For PNe, GALEX's broad-band FUV, NUV fluxes include a mixture of emission lines, varying according to the ionization, and may also include nebular continuum emission (Bianchi et al. 2012). At $\sim 5^{\prime \prime}$ resolution, these data are useful for studies of large objects, where the deep sensitivity, combined with low sky background in FUV, easily reveals faint structures and shock fronts (Fig. 4). In most PNe, the FUV flux is higher than NUV; in some objects (the compact, spherical ones) the FUV and NUV radial light profiles are similar, in others the FUV/NUV ratio varies indicating a complex structure.

\section{Where did the mass go? Atomic and molecular hydrogen}

The ionized shells of $\mathrm{PNe}$ are a wellspring of information, and are spectacular to behold, but most of the progenitor's mass is hiding as non-ionized matter. Typically, the ionized shell and the central star together do not reach one solar mass (e.g. Torres-Peimbert \& Peimbert 1997), but the initial mass may have been up to eight times higher. Molecules are predicted (Ali et al. 2001; Aleman \& Gruenwald 2004), and observed, in the PN environment, surviving even inside the ionized shell (Tenenbaum et al. 2009). $\mathrm{H}_{2}$ emission, and over fifty molecular species have been detected from radio, $\mathrm{mm}$, sub-mm, and IR observations (e.g. Kwok 2003; Chu 2012 and ref. therein, Bujarrabal 2006). The FUSE UV range offers greatest sensitivity to $\mathrm{HI}$ and $\mathrm{H}_{2}$, enclosing a forest of $\mathrm{H}_{2}$ lines from the Werner and Lyman series. The advantage (for observing ISM), and disadvantage (for observing stellar lines), is that this region is extremely sensitive to even tiny amounts of molecular hydrogen. It follows that UV observations shortwards of Ly $\alpha$ become prohibitive along sightlines of high extinction; on the other hand, very small column densities of $\mathrm{H}_{2}$ are easily detectable. In many objects it has also been possible to discern components of different temperature, quite possibly associated with the $\mathrm{PN}$, in addition to the foreground ISM component (Fig. 5). With knowledge, or assumptions, about the geometry distribution, the column density yields the total $\mathrm{H}_{2}$ and $\mathrm{HI}$ (the largest 
portion) mass. Hot ( $\mathrm{T}>2000 \mathrm{~K}) \mathrm{H}_{2}$ was also found in most of the LMC PNe we observed with FUSE and column densities were derived for $\mathrm{HI}$ and $\mathrm{H}_{2}$ (Herald \& Bianchi, 2004a, 2007), as in Galactic objects (Herald \& Bianchi, 2004b, 2002). The gas/dust ratio in the presumably nebular component differs from typical ISM values.

\section{Counting the needles in the haystack: A search for hot CSPN remnants from UV sky surveys}

Large surveys are a paradigm of contemporary astrophysics, owing to several conspiring technological advances: the availability of large, sensitive detectors, data storage becoming cheaper, and browsing of vast databases becoming ever more agile and fast. Recent surveys and homogeneous, cross-connected databases, benefit the field of $\mathrm{PNe}$ as well. A few thousand PNe are known (Parker 2012), and some $10^{4}$ WDs, while $\sim 95 \%$ of all stars are expected to become WD (Kwok 2003), 30-90\% of them going through a PN phase (Drilling \& Schönberner 1985). In addition to their very short life-times, CSPN and their descendants, the hot WDs, are elusive at all wavelengths except the UV, due to their hot $T_{\text {eff }}$ 's to which optical colors are saturated, and faint optical luminosities.

The UV sky surveys by GALEX offer an unprecedented opportunity to detect and characterize the hottest stars (Bianchi et al. 2011a, b, 2007). Out of $\sim 400,000$ sources with FUV-NUV color corresponding to $T_{\text {eff }} \gtrsim 18,000 \mathrm{~K}$, about one tenth have SDSS matches and therefore FUV, NUV, and $u g r i z$ magnitudes are available. Magnitude distributions of these hot WD candidates, analyzed with MW models, place statistical constraints on the initial-final mass relation, favouring low final masses; the result has implications for the yield of chemical elements (Bianchi et al. 2011a). The catalogs of hot WD candidates are available at http://dolomiti.pha.jhu.edu/UVsky and from MAST.

\section{Summary and future directions}

Much progress has been reported in modeling CSPN atmospheres and winds, thanks to critical diagnostics uniquely provided by UV spectra, and improvements in model atmosphere calculations. Wind clumping, soft X-rays from shocks that affect the ionization, and low-abundance ions, were included in recent analyses, which could reconcile previously discrepant diagnostics, and better constrain stellar parameters. Important clues to stellar evolution, such as abundances of other minor elements, await to be extracted even from archival data as more computing power will become accessible, provided that atomic data measurements will also sufficiently progress.

The GALEX UV surveys have turned up an unprecedented census of hot star candidates in the MW, over an order of magnitude larger than previously known hot-WD samples, and with the advantage of the UV-based selection. Towards low-extinction, high Galactic latitudes, GALEX MIS can detect very hot, small stars (e.g. $T_{\text {eff }}=100,000$ $\mathrm{K}, R=0.04 \mathrm{R}_{\odot}$ ) out to $\sim 20 \mathrm{kpc}$, and the scarce halo sample currently known may be improved. Large, more unbiased statistical samples provide tests for stellar evolution theories, and a timely pool of targets for follow-up spectroscopy with upcoming capabilities such as WSO (Shustov et al. 2011) and UVIT, and current HST spectrographs.

Acknowledgments. I am very grateful to the Symposium SOC for the invitation and to the LOC for an impeccable organization, and to G.Keller and J.Herald for help with some figures. Support was provided by the GALEX project and NASA's grant NNX11AI58G.

\section{References}

Güdel, M. 2004, A\& A Rev. 12, 71

Aleman, I. \& Gruenwald, R. 2004, ApJ 607, 865

Ali, A., et al. 2001, MNRAS, 325, 881 
Balick, B. 2012, these proceedings

Bianchi, L. 2011, ApSS, 335, 51

Bianchi, L. 2009, ApSS, 320, 11

Bianchi, L., Manchado, A., \& Forster, K. 2012, these proceedings

Bianchi, L., et al. 2011a, MNRAS, 411, 2770

Bianchi, L., et al. 2011b, ApSS, 335, 161

Bianchi, L., Herald, J., \& Garcia, M. 2009, in Future Directions in Ultraviolet Spectroscopy, $A I P C$, Van Steenberg et al. (eds.), 1135, 145

Bianchi, L., et al. 2008, in Space Astronomy: The UV Window to the Universe, A.I. Gomez de Castro \& N. Brosh (eds.)

Bianchi, L. \& Herald, J. 2007, in The UV Universe: Stars from Birth to Death A.I. Gomez de Castro and M. A. Barstow (eds.), (Editorial Complutense), p. 89

Bianchi, L., et al. 2007, ApJS, 173, 659

Bianchi, L. \& Garcia, M. 2002, ApJ, 581, 610

Bujarrabal, V. 2006, IAU Symp. No. 234, 193

$\mathrm{Chu}$, Y. H. 2012, these proceedings

Drilling \& Schönberner, D. 1985, A\& A, 146, L23

Garcia, M. \& Bianchi, L. 2004, ApJ, 606, 497

Georgiev, L., et al. 2008, ApJ, 681, 333

Hamann, W. R. \& Koesterke, L. 1998, A\& A, 335, 1003

Hamann, W. R., et al. 1998, in: Clumping in Hot Stars Winds: Proceedings of an International Workshop held in Potsdam, Germany, W.R. Hamann, A. Feldmeier, \& L.M Oskinova (eds.), (Universittsverlag Potsdam), p. 75

Hamann, W. R. 2008, ASP Conf. Ser., 391, 293

Herald, J. E. \& Bianchi, L. 2011, MNRAS, in press

Herald, J. E., Bianchi, L., \& Hillier, J. 2005, ApJ, 627, 424

Herald, J. E. \& Bianchi, L. 2007, ApJ, 661, 845

Herald, J. E. \& Bianchi, L. 2004b, ApJ, 609, 378

Herald, J. E. \& Bianchi, L. 2004a, ApJ, 611, 294

Herald, J. E. \& Bianchi, L. 2002, ApJ, 580, 434

Herwig, F. 2005, ARA\&A, 43, 435

Hillier, D. J. \& Miller, D. L. 1999, ApJ, 519, 354

Jahn, D., et al. 2007, A\& A, 462, 281

Keller, G., et al. 2011, MNRAS, in press

Kudritzki, R. Urbaneja, M. A., \& Puls, J. 2006, IAU Symp. No. 234, 119

Kwitter, K. 2012, these proceedings

Kwok, S. 1987, Phys. Reports, 156, 111

Kwok, S. 2003, IAU Symp. No.234, 3

Kwok, S. 2010, PASA, 27, 174

Manchado, A., et al. 2012, these proceedings

Marigo, P., et al. 2001, A\& $\mathcal{G}, 378,958$

Milingo, J.B., et al. 2006,IAU Symp. No. 234, p.459

Oskinova, L., et al. 2007, A\&A, 476, 1331

Parker, Q. 2012, these proceedings

Peimbert, M. 1978, in Y.Terzian ed., IAU Symp. No.76, Dordrecht:Reidel, p.215

Shaw, R. A., et al. 2010, ApJ, 717, 562

Shustov, B., et al. 2011, ApSS, 335, 273

Tenenbaum, E. D., et al. 2009, ApJ, 704, L108

Torres-Peimbert, S., Peimbert 1997, IAU Symp. No. 180, 175

Urbaneja, M. A., et al. 2008, in in: Clumping in Hot Stars Winds: Proceedings of an International Workshop held in Potsdam, Germany, W.R. Hamann, A. Feldmeier, \& L.M Oskinova (eds.), (Universittsverlag Potsdam), p. 67

Werner, K., et al. 2004, A\&SA, 427, 685

Werner, K., et al. 2010, ApJ, 719, 32

Werner, K. 2012, these proceedings 